\title{
From micro- to macro- neutron sources: The Lund Broad-band Neutron Facility
}

\author{
Hanno Perrey ${ }^{1, *}$, Mikael Elfman ${ }^{1}$, Kevin Fissum ${ }^{1}$, Robert J. W. Frost ${ }^{1}$, Nathaly De La Rosa ${ }^{1}$, Markus Kristensson ${ }^{1}$, \\ Per Kristiansson ${ }^{1}$, Nicholai Mauritzson ${ }^{1}$, Francesco Messi ${ }^{1}$, Charlotta Nilsson ${ }^{1}$, Jan Pallon ${ }^{1}$, Emil Rofors ${ }^{1}$ \\ ${ }^{1}$ Lund University, Division of Nuclear Physics, Professorsgatan 1, 22363 Lund, Sweden
}

\begin{abstract}
The Lund Broad-band Neutron Facility provides access to a variety of neutron sources together with a well-established user infrastructure. Neutrons from radioactive sources have been successfully employed for the research and development of both detectors and materials for the European Spallation Source. A recently procured d-t neutron generator delivers higher neutron fluxes than those provided by the radioactive sources, and further allows for pulsed operation. With the currently on-going construction of a dedicated neutron beam-line at our $3 \mathrm{MeV}$ Pelletron accelerator, the facility is anticipated to produce approximately $10^{10} \mathrm{n} / \mathrm{s}$. Cost-effective access to neutrons as well as a platform for educational purposes are the ultimate goals of the project.
\end{abstract}

\section{Introduction}

Neutrons of all energies are important probes of matter and are crucial to an increasingly number of applications in both scientific and industrial fields. For many novel developments and for training purposes, dedicated local facilities offering straightforward and affordable access to neutrons are a vital need of the community. The Lund Broad-band Neutron Facility (LBNF) has been designed with this and a low barrier-of-entry in mind. Hosted by the Division of Nuclear Physics at Lund University and operated by the Neutronics Group, the LBNF provides well-established and user-focused infrastructure, expertise in nuclear physics and detection techniques and access to neutrons from both accelerator-based neutron sources and neutron-emitting radioactive sources. Since 2014 , the facility has functioned as infrastructure for ESSrelated detector and material research and development as well as an educational platform [1]. Currently, the $3 \mathrm{MeV}$ Pelletron accelerator at the LBNF is being upgraded with a dedicated neutron production beam-line. The anticipated increase in neutron flux from approximately $10^{6} \mathrm{n} / \mathrm{s}$ (radioactive source) or $10^{8} \mathrm{n} / \mathrm{s}$ (neutron generator) to $10^{10} \mathrm{n} / \mathrm{s}$ will open up new possibilities for applications in other fields.

\section{Source-based neutrons}

Source-based continuous neutron beams from an ${ }^{241} \mathrm{Am} / \mathrm{Be}$ source provide stable fluxes of typically $10^{6} \mathrm{n} / \mathrm{s}$. At the LBNF, these beams have been successfully used for response characterizations of neutron detectors $[2,3,4]$ and their data-acquisition systems [5]. Using a technique called "tagging" and the infrastructure present at the LBNF, it is even possible to study attenuation and detector responses as function of the neutron energy even with standard radioactive sources $[6,7,8]$. Based on coincidence measurements between prompt gamma-ray emissions and the neutron in the reaction $\alpha+{ }^{9} \mathrm{Be} \rightarrow$
${ }^{12} \mathrm{C}^{*}+\mathrm{n} \rightarrow{ }^{12} \mathrm{C}+\gamma+\mathrm{n}$ which are present in around $55 \%$ of all decays, one can determine the time of flight of the neutron on an event-by-event basis.

As radioactive sources are a stable source of neutrons, they are also a cost-effective tool for educational purposes. At the LBNF, students have used the neutron tagging setup in laboratory exercises in nuclear physics courses. As the setup consists of a water-filled Plexiglas tank, all the key components of the experiment are visible and accessible while keeping the dose-rate on the external surface of the tank well below $0.5 \mu \mathrm{Sv} / \mathrm{h}$. Coupled with modern teaching techniques such as flipped classroom and blended learning, the lab makes key concepts such neutron interactions in matter, neutron detection and neutron time of flight tangible and thus facilitates learning. Figure 1 shows a photograph taken during one of the lab exercises where students test the effect of shielding materials on the measured neutron flux.

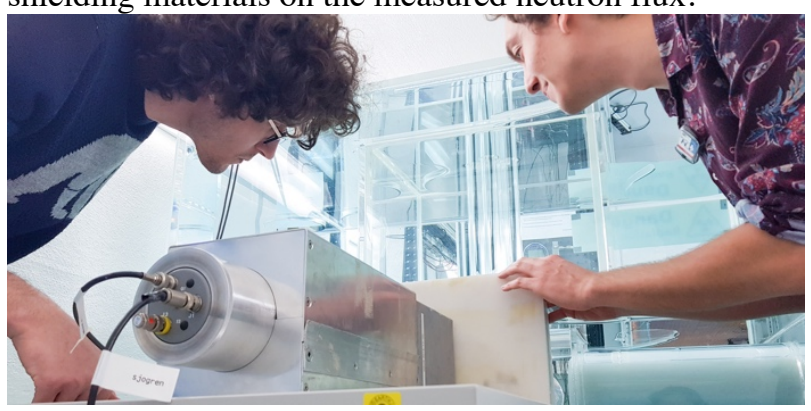

Figure 1: Photograph taken during neutron time-of-flight lab exercise showing students interacting with the setup. Visible are the water-filled tank hosting the radioactive source (background), the neutron detector (foreground) and shielding material being introduced into the neutron beam.

\section{Accelerator-based neutrons}

For applications requiring higher or variable neutron fluxes, the LBNF features a SONDERN Genie 16 neutron generator. Based on the $\mathrm{d}+\mathrm{t}$ reaction, calibrated fluxes of up to $10^{8} \mathrm{n} / \mathrm{s}$ can be achieved in DC operation mode. Even

\footnotetext{
* Corresponding author: hanno.perrey@nuclear.lu.se
} 
higher fluxes are anticipated from the currently ongoing upgrade of the $3 \mathrm{MeV}$ Pelletron accelerator with a dedicated beam-line for neutron production. A $10 \mu \mathrm{A}$, $2.5 \mathrm{MeV}$ deuterium beam will be directed onto a beryllium target which, when run in continuous mode, is anticipated to produce approximately $10^{10} \mathrm{n} / \mathrm{s}$ in $4 \pi \mathrm{sr}$.

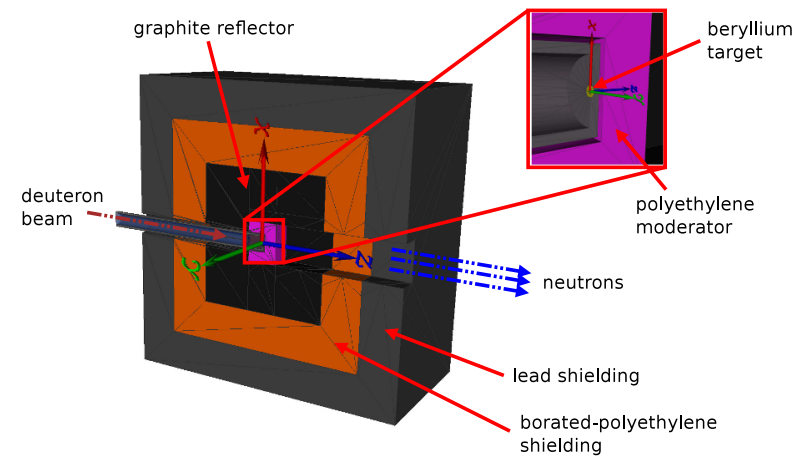

Figure 2: Geometry of the moderator and shielding assembly as simulated in GEANT4

A polyethylene moderator, graphite reflector and lead/borated-polyethylene shielding system will then thermalize the neutrons and direct them to the experimental area. The design for the moderator/shielding assembly is illustrated in Figure 2. The functionality of each of its material layers are as follows: The innermost polyethylene block, which surrounds the end of the beamline, moderates fast neutrons exiting the beryllium target. From this moderator block there is an exit path cut through the surrounding layers in the forward direction to allow neutrons to escape. Surrounding the moderator is a graphite layer which acts as a neutron reflector, redirecting neutrons that escape the moderator in all but the forward direction. The graphite layer is then surrounded by borated-polyethylene to capture escaping neutrons that are not fully reflected. Finally, an outer casing of lead is placed to absorb gamma-rays produced in the target and by thermal neutron capture on boron.

At present the beam-line required to transport the deuterons, including all magnets, the electrostatic deflector system, a beam-dump and water-cooled targetholder, has been constructed and is ready for operation. Extensive simulations have been performed in GEANT4 [9] to model the performance of the moderator and shielding assembly.

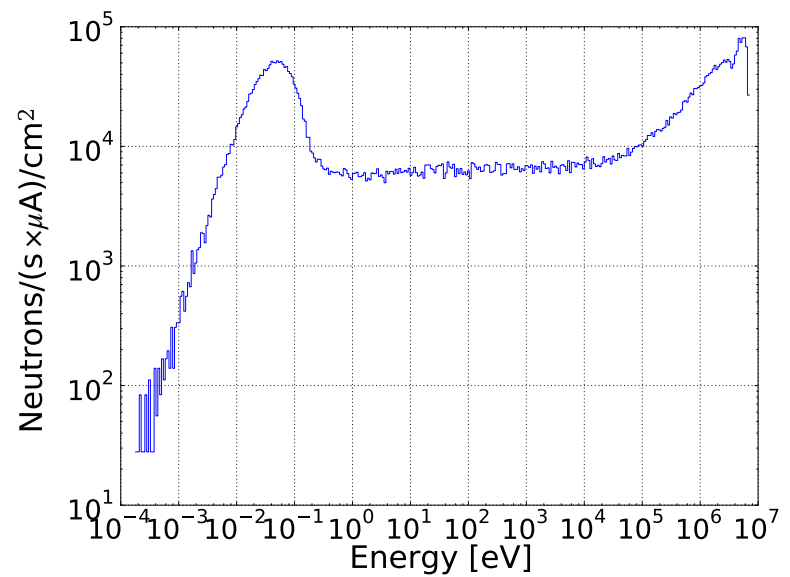

Figure 3: Simulated neutron energy spectrum at the exit port of the current moderator/shielding concept.

With the deuteron beam run in continuous mode the neutron flux at the exit port in the moderator/shielding assembly is anticipated to be on the order of $5 \times 10^{5}$ $\mathrm{n} / \mathrm{cm}^{2} / \mathrm{s}$, of which the thermalized-neutron flux will be around $5 \times 10^{4} \mathrm{n} / \mathrm{cm}^{2} / \mathrm{s}$. Figure 3 shows the simulated neutron flux per $\mu \mathrm{A}$ of deuteron beam as function of neutron energy directly at the exit port of the currently favoured moderator concept. The spectrum shows a peak at neutron energies between $20-100 \mathrm{meV}$ and contributions of similar order of magnitude from fast $(>1 \mathrm{MeV})$ as well as neutrons of intermediate energies. Further control of the neutron energy will be implemented by pulsing the beam using an electrostatic deflector and using time of flight to discriminate against unwanted neutron energies. Such an increase in neutron flux compared to the other sources available at the LBNF opens up many possibilities for neutron-detector characterizations or fast-neutron activation analysis targeting fast decay activation products using the available high-sensitivity gamma-ray spectrometer. Further experimental instrumentation at the neutron beam-line is foreseen to be developed and depends on future users' and collaborators' needs.

\section{Summary}

The LBNF provides its users with a variety of options for neutron sources of different intensities and beam characteristics. An on-going upgrade of the Pelletron accelerator with a dedicated neutron beam-line will extend this suite of sources and is expected to produce approximately $10^{10} \mathrm{n} / \mathrm{s}$.

This cost-effective access to a wide range of neutron energies and beam types reduces the barrier of entry to novel neutron-related developments and applications in both science and industry significantly. Furthermore, such a facility located at a University provides an ideal site for the education of the next generation of neutron scientists.

\section{References}

1. Messi, Perrey et al, accepted by IAEA (2018) 
2. Issa, Khaplanov, Hall-Wilton, Llamas, Riktor, Brattheim \& Perrey, Phys. Rev. Accel. Beams, 20, 092801 (2017). doi.

3. Mauri et al, Journal of Instrumentation, 13(03), P03004-P03004 (2018). doi.

4. Messi, Mauri \& Piscitelli, NIM A, 936, 499-500 (2019). doi.

5. Christensen, et al, Journal of Instrumentation, 13(11), (2018). doi.

6. Scherzinger, et al, NIM A, 840, 121 - 127 (2016). doi.

7. Scherzinger et al, Appl. Rad. and Isotopes, 128(C), 270 - 274 (2017). doi.

8. DiJulio, Cooper-Jensen, Perrey, Fissum, Rofors, Scherzinger \& Bentley, NIM A, 859, 41 - 46 (2017). doi.

9. J. Allison et al, NIM A, 835 186-225 (2016). doi 